\title{
DLR's Advanced Telerobotic Concepts and Experiments for On-Orbit Servicing
}

\author{
Detlef Reintsema $^{1}$, Klaus Landzettel ${ }^{2}$, and Gerd Hirzinger ${ }^{3}$ \\ Deutsches Zentrum für Luft- und Raumfahrt e.V. (DLR) \\ in der Helmholtzgemeinschaft \\ Institut für Robotik und Mechatronik \\ ${ }^{1}$ Detlef.Reintsema@dlr.de \\ 2 Klaus.Landzettel@dlr.de \\ ${ }^{3}$ Gerd.Hirzinger@dlr.de
}

Summary. Space robotics will become a key technology for the exploration of outer space and the operation and maintenance of space stations, satellites and other platforms, saving costs and relieving man from dangerous tasks. But we do not have to wait until robots are really autonomous or intelligent, since by modern tele-operation and tele-presence we are able to remotely control robot systems from the ground in the sense of "prolonging man's arm into space". Humans, with their several hundred thousand years of evolution, will not adapt themselves to the hostile space environment, whilst robots, which have only been developed for just over 40 years, can be much more easily adapted to such an environment. As presented within this work few pioneering telerobotic experiments like ROTEX, the first remotely controlled space robot system, ETS-VII, the first free-floating space robot experiment, or ROKVISS, Germany's recent advanced space robot experiment on the International Space Station, have been proposed and conducted on the way towards a space robot assistant system for the usage as an artificial astronaut to perform On-Orbit Servicing (OOS) tasks.

\section{Introduction}

Although there exists a large application field for telerobotics in space the ongoing worldwide telerobotic activities concentrates on three specific mission and application fields: on-orbit assembly and servicing, (science) payload tending and planetary surface robotics. Within these fields of application the advanced robotics tasks to be handled range from preparation and operational support of scientific experiments (both internal and external) on a space station, over inspection, maintenance, and repair of orbital infrastructure and 
systems, towards recovery and cleaning up of space debrise, as well as exploration and coverage of planets and orbs in general.

In general, the design and deployment of space robotic components is caused by the specific manipulation and mobility aspects of the mission's requirements. For instance, the increasing number of launched satellites per year calls for solutions

- to overcome malfunctions or system failures and keep space infrastructure operational, as well as extend the operational lifetime of ageing space systems and satellites like stations or telecommunication systems.

- to keep free operational space for telecommunication systems in geosynchronized orbit, as well as to avoid the endangering of space systems in LEO and of the public living in the habited parts on Earth. Examples for such dangerous stranded space systems in the past are Skylab and MIR. In the future, the uncontrolled and accidental de-orbiting of other huge satellites is expected, where parts of these will hit the surface of the Earth.

Robotics scientists are developing tele-operated human-like robots for orbital servicing (On-Orbit-Servicing, OSS), to potentially eliminate the need for dangerous and expensive astronaut servicing. As the following sections will show, nowadays robotics technology is mature enough to act as a useful tool in supporting the astronaut during useful work. In addition, advanced robotics technology can relieve the human from servicing tasks to perform tasks best suited to human decision-making and flexibility that is unlikely in the near-term to be matched by autonomous or tele-operated robots. DLR's achievements on advanced telerobotics concepts in On-Orbit-Servicing within the last 20 years give an impression about the near future space robotics capabilities in case of On-Orbit-Servicing (see Sec. 4).

\section{The Space Robotics On-Orbit Servicing Profile}

When comparing human skills with those of present-day robots of course human beings in general are by far superior, but when comparing the skill of an astronaut in a clumsy space-suit with that of the best available robot technology, then the differences are becoming smaller. For intra-vehicular laboratory activities (IVA) e.g. a robot basically would have to compare with the full human skill and mobility. To be honest, many of the manual operations to be done in a space-laboratory environment are fairly simple standard operations, like handling parts, opening and closing doors, pulling drawers, pushing buttons etc. which have to be done just by stepping through extensive, written procedures. Real intuition and manual skill is particularly requested in nonnominal situation and repair situations. Although it is not clear today when a multi-fingered robot hand might be as skilled as the human hand and when (if ever) a robot might show up real intelligence and autonomy, it nevertheless is 

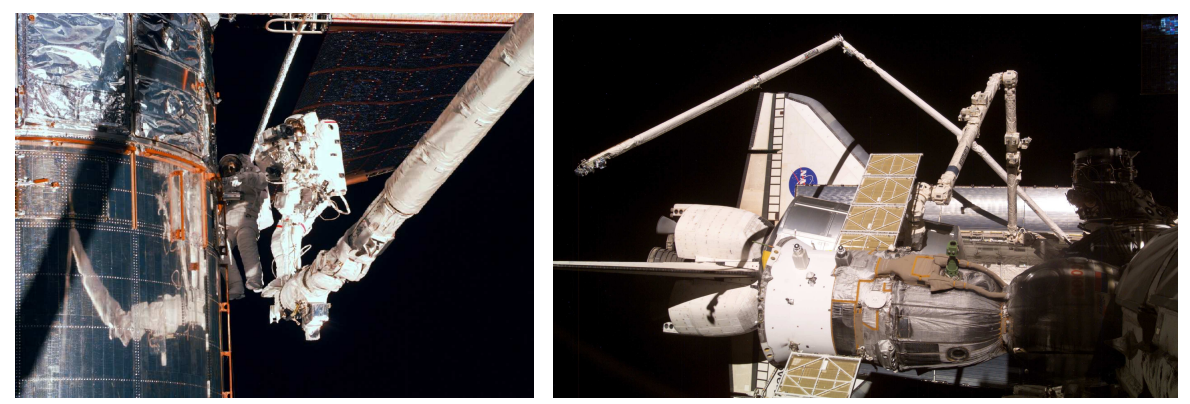

Fig. 1. Typical manipulation profiles to serve for by astronauts are associated with the assembly of space structures or satellite servicing missions as maintenance of the Hubble Space Telescope (left). In general, most of the servicing technology has been pushed from the International Space Station (ISS) programme. A number of cargo-handling manipulators are proposed to operate on the ISS as the European Robotic Arm (ERA), the Japanese Remote Manipulator System (JEMRMS), and even the US Mobile Servicing System (MSS) including the Space Station Remote Manipulator System (SSRMS, Canadarm2) (right). [Photos by courtesy of NASA]

obvious that even with today's technology and the available telerobotic concepts based on close co-operation between man (e.g. the ground operator) and machine, there are many tasks in space, where robots can replace or at least augment human activities with reduced cost from a mid-term perspective.

Launch access to orbit, orbital and target inspection maneuvering are mandatory before a robot can serve for proximity operations like in-orbit assembly, maintenance of equipment, and replenishment of consumables towards system upgrade and repair (see Fig. 1) [4]. In general, robot servicing can be achieved through the exchange of modular components (Orbit Replaceable Units, ORU); To minimize the complexity of a servicing task ORUs are widely in use within space to provide an easy module-based replacement concept to serve for. Major maintenance tasks are the re-supply of consumables (fluids, materiel and data storage) to the target and cleaning/resurfacing/decontamination charging. Diagnoses and correction of faults or failures are typically tailored by ORUs examination and replacement.

The first general purpose robotic manipulator ${ }^{4}$ designed for specific use in the harsh environment of space was the Shuttle Remote Manipulator System (SRMS, Canadarm). SRMS has successfully flown on over 70 Shuttle flights and has retrieved more than a dozen satellites [3]. The SRMS was the first robotic manipulator to demonstrate the principle of robotic servicing. The Canadarm2 and the original Canadarm (see Fig. 1, right side) could work together, handing payloads to one another.

\footnotetext{
4 "Manipulator" and "robot arm" have similar concepts. "Manipulator" means a machine similar to a human arm that can do various operation, whereas "robot arm" has a nuance of an arm of a "robot" with certain degree of intelligence.
} 

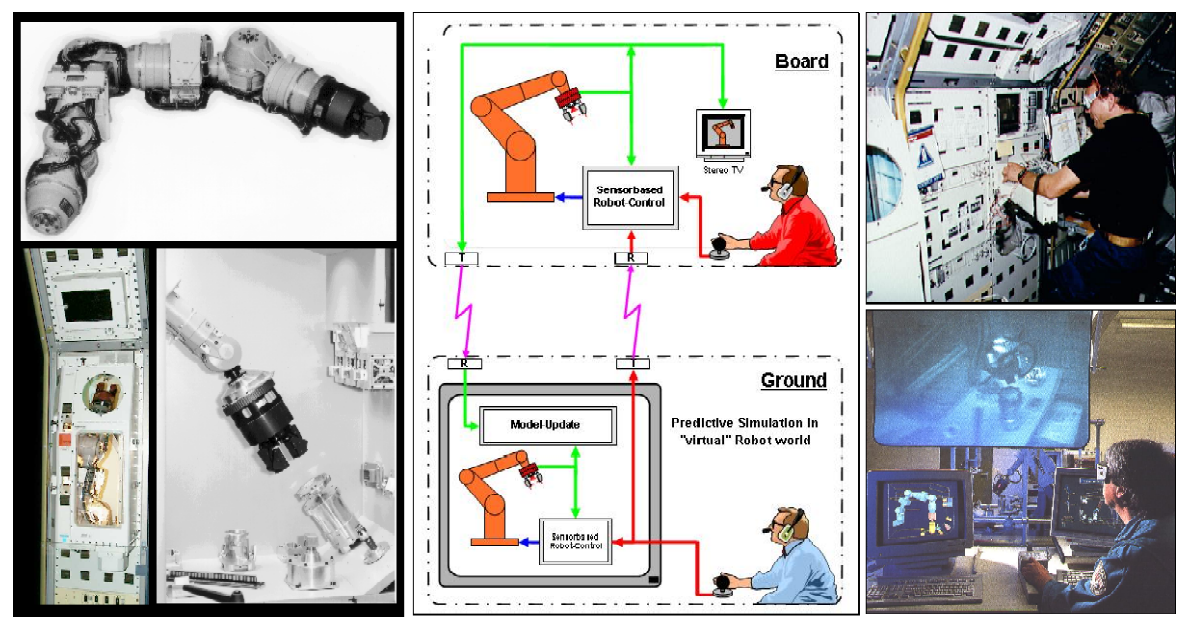

Fig. 2. The first remotely controlled robot in space, a multi-sensory robot on board of a shuttle worked in autonomous modes, teleoperated by astronauts, as well as in different telerobotic control modes by a human operator from ground.

\section{Pioneering Orbital Telerobotics Experiments}

Future On-Orbit-Servicing systems seems to be designed as unmanned spacecrafts which will be remotely operated by a human operator on ground or an astronaut on board of an orbiter spacecraft or space station. In spite of the fact that all these proposed new systems are still in the design or preparation phase a lot of know-how concerning the operation and the design of space robot servicer systems was gained during previous space robotics missions. Within the last 20 years three pioneering space robot experiments have been performed with the aim on demonstrating key robotics technology for unmanned On-Orbit-Servicing activities: ROTEX (1993) - The first remotely controlled space robot system, ETS-VII (1998) - The first free-flying space robot system, and ROKVISS (2005) - The first high-fidelity telepresence controlled high performance light-weight robot on the ISS.

\subsection{ROTEX - The first remotely controlled space robot system}

With the Spacelab-Mission D2, flown at the end of April 1993, the first remotely controlled robot arm was carried into the earth orbit inside the cargo of the Shuttle COLUMBIA. The advanced Space Robot Technology Experiment ROTEX was the begin of Germany's participation in space automation and robotics. For the first time in the history of space flight a small, multi-sensory robot proposed and realized by DLR's Institute of Robotics and Mechatronics (DLR-RM) have been conducted to demonstrate the feasibility of servicing 


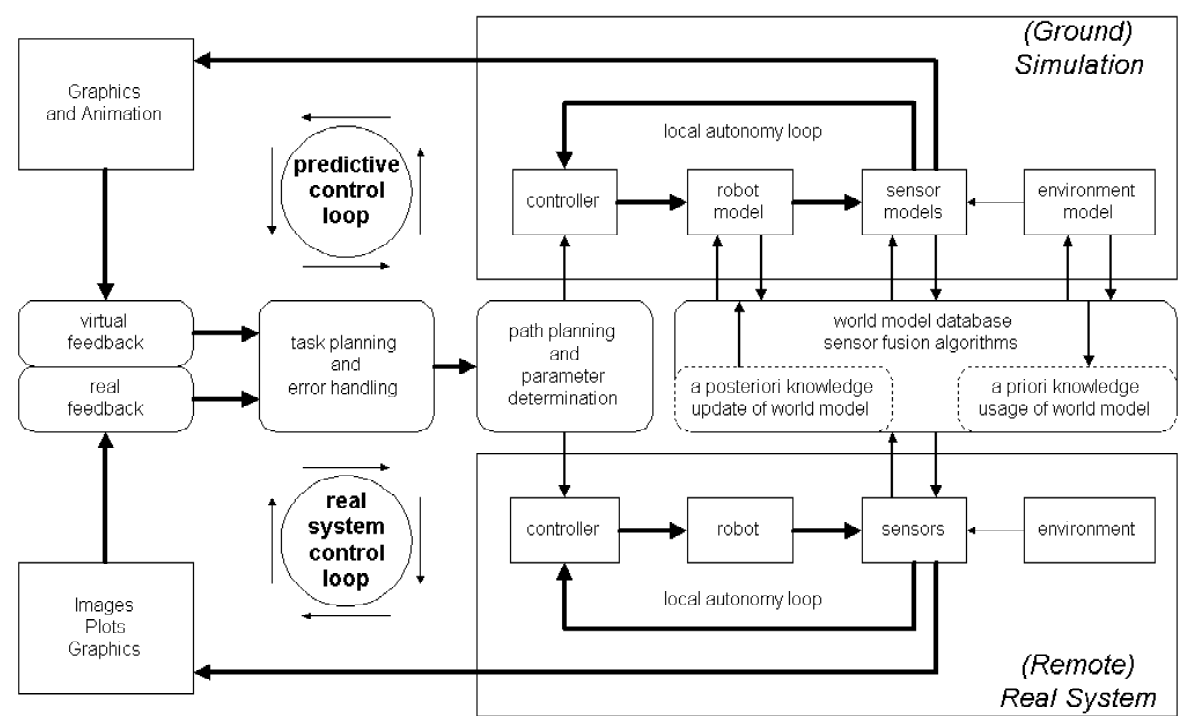

Fig. 3. The telerobotic concepts of ROTEX: shared local autonomy, shared control and optional switching between task level, sensor-based teleprogramming and online-teleoperation using predictive graphics (see also Fig. 12).

prototype capabilities by a remotely controlled robot inside the SpacelabModule. The basic goals of ROTEX have been (1) the verification of joint control (including friction motion) under zero gravity, (2) the evaluation of DLR's sensor-based 6 DoF hand-controller (one-hand operation) under zero gravity, and (3) the performance demonstration of a complex, multi-sensory robot system with powerful man-machine-interfaces, in a variety of operational modes, including on-line teleoperation and off-line programming from ground (see Fig. 3) [11].

ROTEX has been operated within an enclosed workcell integrated into a rack of the Spacelab-D2 Module (left side of Fig. 2). Key operational modes for the remote robot control have been performed from both, an on-board workstation integrated into another rack and from DLR's ground control center in Oberpfaffenhofen (right side of Fig. 2). The defined workcell was prepared to demonstrate the most different applications by not restricting the performed prototype tasks to internal servicing operations, but also aiming at assembly and external servicing (e.g. grasping a floating satellite) as assembling a mechanical truss structure from identical cube-link parts, connecting/disconnecting an electrical plug, to simulate an ORU exchange using a bajonet closure mechanism, and grasping a floating object.

The ROTEX manipulator was a small robot arm built up by six joints to be able to reach in all directions and grasp objects within the enclosed workcell. A multi-sensory gripper has been provided a number of sensors including 

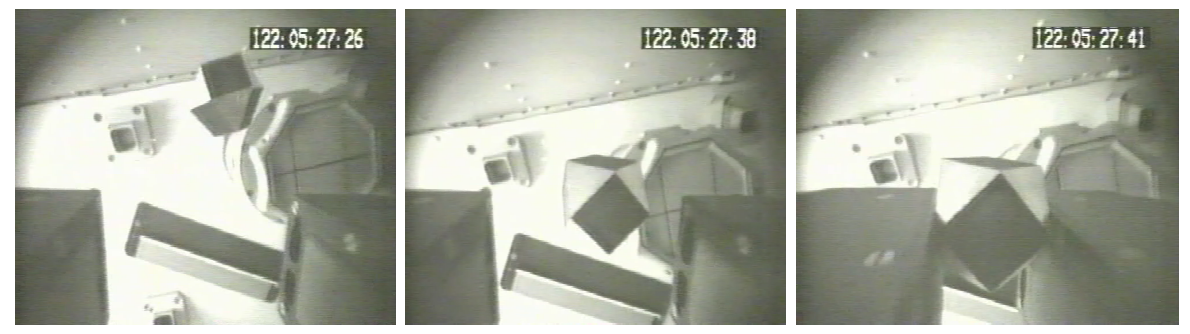

Fig. 4. One of the pioneering experiments in ROTEX has been the fully automatic grasping of a free-flying object from ground despite a varying round-trip-delay of up to 7 seconds.

two 6-axis force-torque wrist sensors located at the back of the gripper to prevent the robot of overloading, a gripping assembly containing an array of 9 laser for distance-measuring, tactile sensors and grasping force control. A tiny pair of stereo television cameras within the gripping assembly gave a direct view of the manipulated object. In addition a fixed pair of video cameras provided stereo images of the whole workcell.

The basic operational modes of ROTEX have been based on a unified shared local autonomy control concept that distributes intelligence between the operator and the teleoperator in the sense of a task-directed approach, termed as tele-sensor-programming (TSP) approach (compare Fig. 3) [13, 8]. Presuming that sufficient information about the actual environment is available from sensors, partial tasks can be executed independently on the machine level. Local sensory feedback control loops are executed by the robot system, while global task planning has to be done interactively by a human operator (shared local autonomy approach). To provide ROTEX with the capability of acting and reacting in such an autonomous way the TSP concept has been based on a sufficient shared control approach $[2,1]$. Herein, the control of the operation is shared between local sensory feedback control loops, closed at the robot's site (i.e. on-board and in the predictive ground simulation), and gross commands, which are generated by the robot control system of the operator. Basic or gross commands are refined autonomously providing the robot with a modest kind of sensory intelligence [33], and may be originated from a human operator handling the control/sensor ball or alternatively from an intelligent path planning module [12].

Even if the essential telerobotic control concept of ROTEX has used the integrated sensors, on-board sensory feedback was restricted to force-torque and range finder signals only due to on-board processor limitations. In general, feedback to the human operator during on-line teleoperation was provided via the visual system (stereo video images). In addition, to deal with time delays of up to 5-7 seconds, a powerful delay-compensating predictive 3D-stereographic simulation of the robot and the workcell has been provided for the operator on ground, which has included the robot's sensory behavior. 

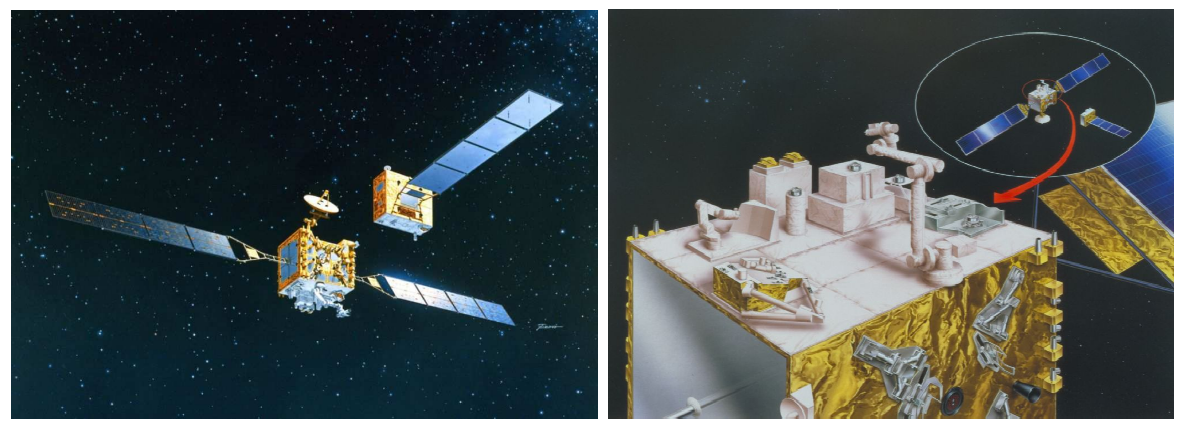

Fig. 5. The ETS-VII satellite was designed to verify key technologies on autonomous rendezvous, docking (left) and robotics technologies in space (right). To experiment the rendezvous-and-docking maneuver HIKOBOSHI has opened the docking mechanism holding ORIHIME and started to separate from each other at the low speed of $2 \mathrm{~cm} / \mathrm{sec}$. After separation HIKOBOSHI and ORIHIME flew in formation for several minutes at a constant distance. On command HIKOBOSHI started approaching ORIHIME along the flight direction $(1 \mathrm{~cm} / \mathrm{sec})$ and captured ORIHIME with the docking mechanism [?]. [Photos by courtesy of JAXA]

The most considered experiment was the autonomous catching of a freefloating object6 (see Fig. 4). This experiment may be treated as a precursor mission to the long term goal of capturing a non cooperative, tumbling satellite in free space (see also TECSAS 4.2). Due to the lack of space proven image processing hardware, for the capturing experiment the control loop was closed via an image processing system on ground. The current total signal round trip time was determined during experiment execution and regarded by the prediction of the free floating part's position. This principle may be directly applied for today's satellite repair missions in order to keep the required hardware effort for the space craft as low as possible $[12,15]$.

\subsection{ETS-VII Satellite - The first free-floating space robot}

In November 1997, the 7th satellite of the ETS satellite series, proposed and realized by the Japanese space agency JAXA (formerly NASDA), was launched. The ETS-VII satellite was designed to verify key technologies on autonomous rendezvous, docking and robotics technologies in space $[41,49,50]$. To demonstrate an autonomous rendezvous and docking maneuver the ETS-VII system (see Fig. 5, left) has been composed of two unmanned spacecrafts, a servicer satellite (Hikoboshi) and a smaller client subsatellite (Orihime). The first free-floating space robot arm was mounted on an Earth pointing surface of the servicer satellite to perform several teleoperation experiments.

The robotics experiment system of ETS-VII (see Fig. 5, right) has been composed by a 6 DoF robot arm and various payloads designed for prototypical tele-operated experiments like manipulating a simulated ORU [43, 42, 47]. 
To handle payloads like small equipments or to grasp a floating object like the target satellite specific tools as the taskboard or the target satellite handling tool have been used. Up to six CCD cameras were installed on board to monitor the ETS-VII system, especially the telerobotics experiments. A pair of hand cameras were used as an on-board vision sensor for visual servicing control of the robot arm.

The ETS-VII robot arm has been provided three kinds of control modes: a joint angle/velocity control mode, a compliance control mode (including force control, active limp and impedance control) and an arm tip position/attitude control mode. To evaluate the performance of the robot's compliance control capabilities a peg-in-hole task has been experimented. Occurred errors in position and attitude of the peg were corrected by the compliance controller, in particular a local sensory feedback control loop approach as within ROTEX.

A time delay of approximately seven seconds for ground controlled teleoperation requests telerobotic concepts like shared control, predictive display and visual guidance as introduced with ROTEX. Thus, it is obvious to use similar concepts within ETS-VII and to distinguish between an automatic tele-programming mode and a telemanipulation mode to command and operate the robot arm from ground. In general, in case of telemanipulation the robot arm was manually controlled by a set of joysticks, a translation and a rotation hand controller.

The advanced telerobotics experiments of ETS-VII have been demonstrated the first ground-space bilateral teleoperation limited due to a large time delay [51, 50, 52, 44]. Several experiments as a slope tracing task and a peg-in-hole task were tele-operated by a model-based bilateral control approach using a 6 DoF haptic interface as master. Task performance was compared between the bilateral mode and the unilateral mode with force telemetry data visually displayed on a screen. As experienced with the ETS-VII system, kinesthetic force feedback to the operator is helpful even under such a long time delay, to improve the performance of the task.

\subsection{GETEX - The German ETS-VII Technology Experiment}

The invitation to participate in the Japanese ETS-VII project led up to the verification of the dynamical considerations and lab experiments of the nationally funded Experimental Servicing Satellite (ESS) study under real mission conditions (see Fig. 6). In April 1999 DLR-RM received the permission from our Japanese colleagues to remotely program and control their robot from Tsukuba/Japan [24]. The so-called GETEX (GErman Technology EXperiment) project, performed in cooperation with the Institute of Robotics Research (IRF) at the University of Dortmund [16], was very successful as was the whole ETS-VII mission. The ETS-VII robot has been operated

- to verify the dynamic models for the interaction between a robot arm and its free-flying carrier [19]. The results gained from this crucial experiment represent an important step towards a free-flying service satellite [22, 21]. 

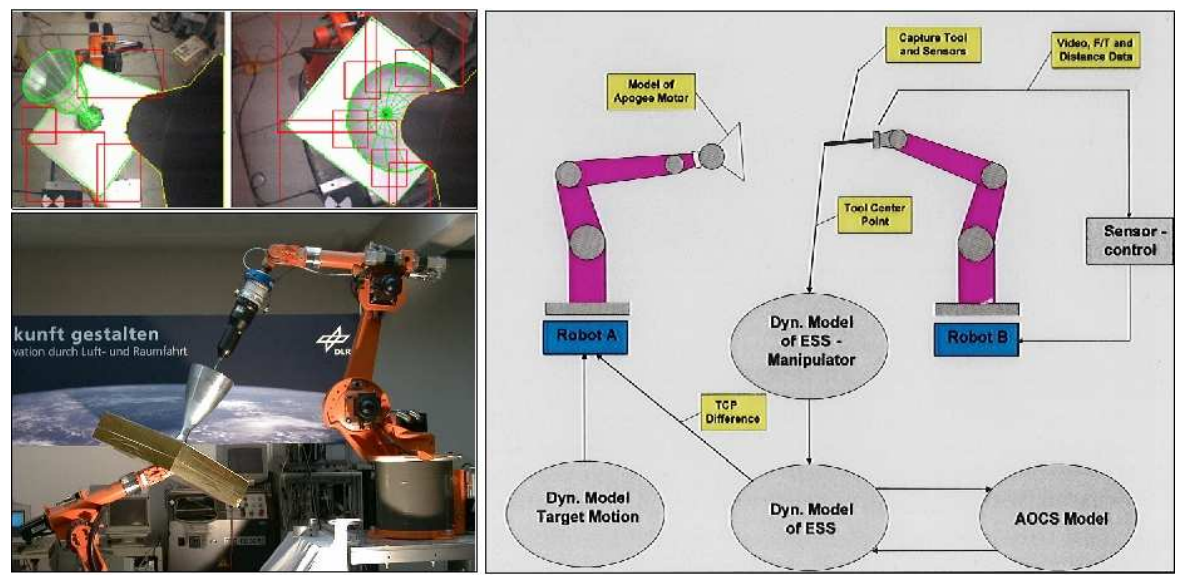

Fig. 6. In the course of the (ESS) study, DLR-RM has been investigated the dynamical behavior of a free-flying servicing satellite (carrying a robot arm) to find out the influence of the robot arm motion to the pose (position \& orientation) of the servicer satellite [26, 37, 27]. A hardware-in-the-loop (HIL) simulator, which mainly consist of a two-robot system and the relevant dynamic models of the involved subsystems, was developed allowing the simulation of the mission critical final approach and capturing phases, taking the robot's motion into consideration.

- to verify our sensor-based task-level programming approach including onboard autonomy via selected image features and force-torque information; - to use our world model update approach using real video images from space and relating them with virtual images from the 3D CAD model.

To gain a reasonable amount of experimental data for the dynamics verification, the major part of the GETEX experiment time was allocated to dynamic motion experiments, which consisted of a series of motions carried out by the manipulator while the attitude control system of the ETS-VII carrier was switched off.

In general, if a robot which is mounted on a spacecraft moves, it generates linear and angular momentum. The attitude control system will permanently produce forces and torques compensating for the arm motion disturbances. The spacecraft may then be considered as inertial in the co-ordinates of an orbit-fixed system, and the problem of robot motion planning can be solved using the same methods as for terrestrial, fix base manipulators. Due to the linear momentum conservation, which states that the center of mass of the system comprising the robot and the satellite is constant, the motion of a manipulator mounted on the satellite will lead to a compensating motion of the satellite. The amount of satellite translation produced, depends on the masses of the bodies constituting the system. 

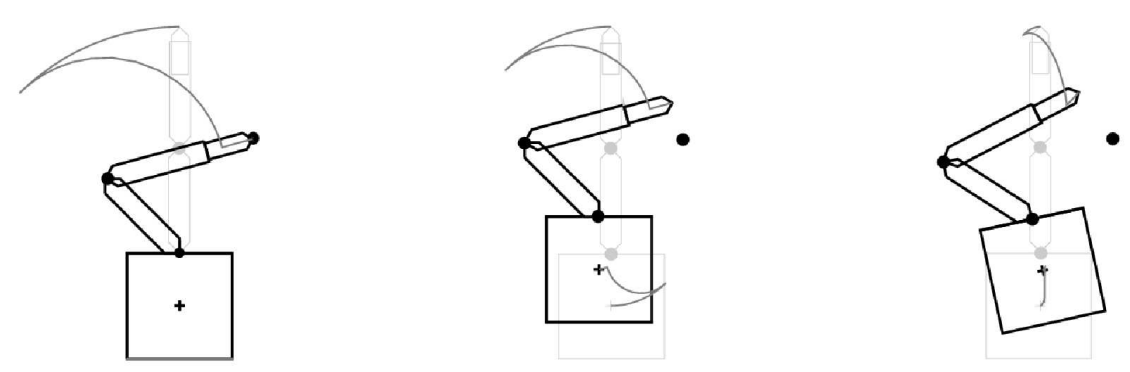

Fig. 7. The influence of the satellite attitude control mode on the path described by the robot end-effector - the same joint motion is carried out by a robot with a fixed base (left), an attitude controlled robot (middle) and a free-floating robot (right).

For space robotic systems which are neither position nor attitude controlled the angular momentum conservation law leads further to a rotation of the spacecraft, by an amount which results from the mass and inertia properties of the manipulator links and the spacecraft. It is generally assumed that no external forces act on such free-floating robots [38, 39]. The free-floating mode of operation is of interest for space robots not only for the reason that attitude control fuel may be saved, it will also be of importance during repair missions, when the servicing satellite is very close or in contact to the target satellite: any action of the attitude control system of either of the two satellites during this phase could lead to a collision and thus to a potential damage on the two spacecrafts.

As long as the tasks performed with the robot are described in robot-fixed coordinates, the fact that the satellite position remains uncontrolled has no influence. If, however, the task is described with respect to an orbit-fixed coordinate system, as it would be the case for example for the capturing of a defect satellite, the satellite's motion has to be taken into account (see Fig. 7).

\subsection{ROKVISS - High performance light-weight robotics in space}

Space presents an extremely harsh operational environment for robotics technology that limits the on-orbit life and performance capability of space robotics technologies, in particular the electronics components, due to radiation surroundings. The break for (low-cost) intelligent space robotics technology has been the absence of innovative, high-performance, and survivable electronics space components. Even if radiation-hardened (rad-hard) circuit versions are available their tolerance levels are not always compatible with the Space requirements. So far, technology for space robotics applications has to deal with bulky and most expensive rad-hard components which limits the performance and capability of space robots, especially of telerobotics applications in space. The ongoing telerobotics experiment ROKVISS - Robotics 


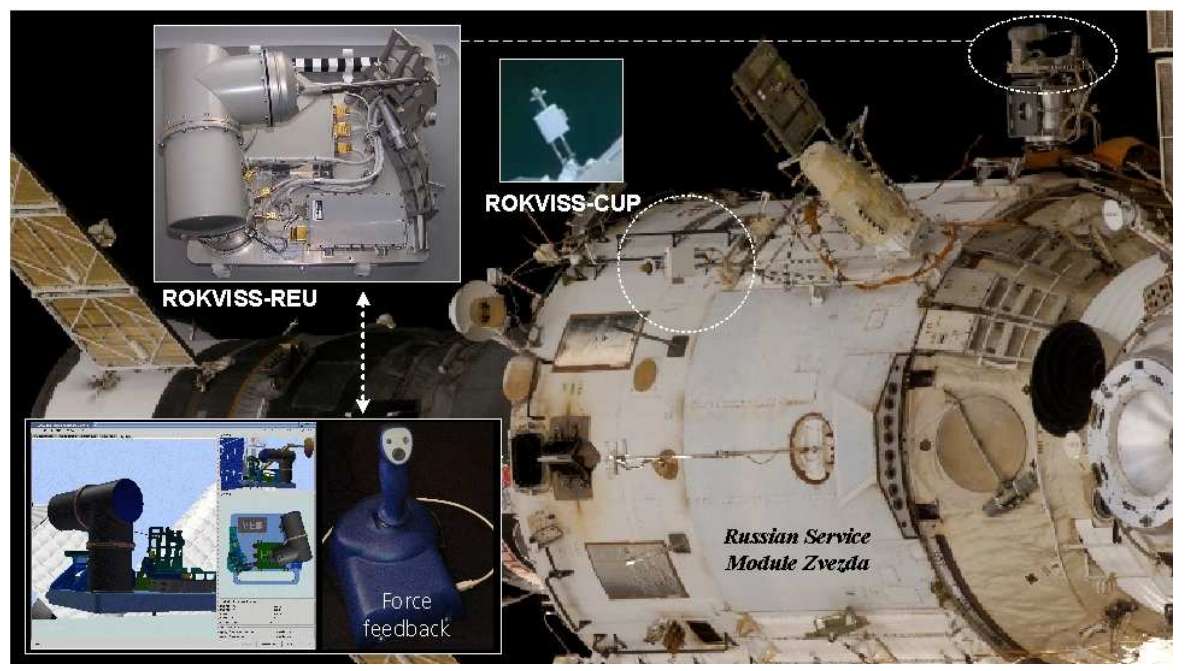

Fig. 8. At the end of a 5 hours space walk the astronauts succeeded in mounting the external flight unit (REU) and S-Band antenna (CUP) of ROKVISS, and connecting the necessary cables to the on-board controller. [ISS-Photo by courtesy of NASA]

Component Verification on the International Space Station (ISS) - has been prepared to demonstrate the feasibility of innovative, high-performance, and survivable electronics components for a new kind of intelligent robotics joint technology as required for On-Orbit-Servicing applications.

ROKVISS has been proposed to verify and demonstrate the performance and capabilities of DLR's modular light-weight, torque-controlled robotic joints under real space conditions [17]. Composed by a lot of common electronics components, which are identical to those used in DLR's seven joint light weight robot [34, 31], a latch-up protection power supply circuit has been designed and integrated into the robotics joint electronics to prevent burn out of the robotics joint hit, and hence to protect the integrated sensors and common industrial electronics components. Thus, ROKVISS investigates the functions and capabilities of robotic joints, and will identify their dynamic and friction behavior over a long time duration, with the aim to get rid of bulky and most expensive rad-hard components for space application in favor of highly integrated circuits [17].

ROKVISS is the second space robot mission after ROTEX proposed by DLR's Institute of Robotics and Mechatronics ${ }^{5}$. During a space walk in January 2005 the external flight unit (REU) and a dedicated S-Band antenna

\footnotetext{
${ }^{5}$ ROKVISS was realized by DLR's Institute of Robotics and Mechatronics (DLR$\mathrm{RM}$ ) in cooperation with the German space companies EADS-ST, Kaiser-Threde, and vHS (von Hörner \& Sulger) and close collaboration of the Russian Federal Space Agency ROSKOSMOS and RKK Energia.
} 
have been mounted outside at Zvezda, the Russian Service Module of the ISS (Fig 8). Inside the service module CUP was connected to the on-board controller (OBC) of the REU. The REU of ROKVISS (see right-hand side of Fig. 10) is composed by a small robot arm with two torque-controlled joints, a power supply, and a video supply to control a stereo camera, an earth observation camera, and an illumination system. A mechanical contour device is placed within the working environment of the robot arm to verify the robot's functions and performance. Since February 2005, ROKVISS is operated by DLR-RM in close collaboration with ZUP, the ISS ground control station in Moscow. After one year of successful operation the ROKVISS hardware is still working, and the mission will be prolongated for another year.

For the first time in space robotics a direct radio link contact between on-ground and on-board controllers is used for ground-controlled teleoperation instead of a delayed inter-orbit communication link as realized within ROTEX or ETS-VII, who have been used a tracking and data relay satellite $[11,41]$. Thus, the OBC of ROKVISS has direct access to CUP, a dedicated S-Band communication system including a separate boom antenna pointing to the earth (Fig 8). On earth the ROKVISS ground controller (OGC) has also direct access to a transceiver system (Cortex Data EGSE) of DLR's tracking station in Weilheim [17]. To get access to the OBC the direct radio link must be established before. Using a direct radio link limits the access time to the on-board system down to time windows of 8 minutes length, when the ISS passes through the tracking space of the ground antenna.

Deviant of past space robotics experiments the ROKVISS communication protocol is served by OBC/OGC instead of using external support equipment. Both are responsible for (de-)modulation of ROKVISS-data into frames which are transferred over the S-Band channel. In spite of ESA requirements the transfer frame protocol is compliant to the CCSDS telemetry/telecommand standards which have been tailored as described in [17] to get rid of protocol overhead, and to meet the specific real-time requirements of the telepresence mode (a proposed sample rate for transfer of $500 \mathrm{~Hz}$ and a jitter of at most $1 \mathrm{~ms}$ ). In general, the dedicated S-band communication link provides an overall data rate of $256 \mathrm{kbit} / \mathrm{s}$ for telecommand (uplink-channel) and $4 \mathrm{Mbit} / \mathrm{s}$ for telemetry (downlink-channel) data, including 3,5 Mbit/s for videodata. Due to a lean, dedicated point-to-point protocol implementation the measured round-trip times are less than $20 \mathrm{~ms}$ depending on the position of the ISS.

During one year of operation the robot joints have been extensively tested and identified (dynamics, joint parameters) by repetitively performing predefined robot tasks in an automatic mode, or even by direct operator interaction. For the design of the joint level controller (position, torque, and impedance control capabilities) efficient and reliable autonomous off-line identification methods have been developed, which allow the stiffness, damping and friction parameter identification at joint level. Starting from the model and corresponding identification measurements, a modified time-efficient, on-line iden- 

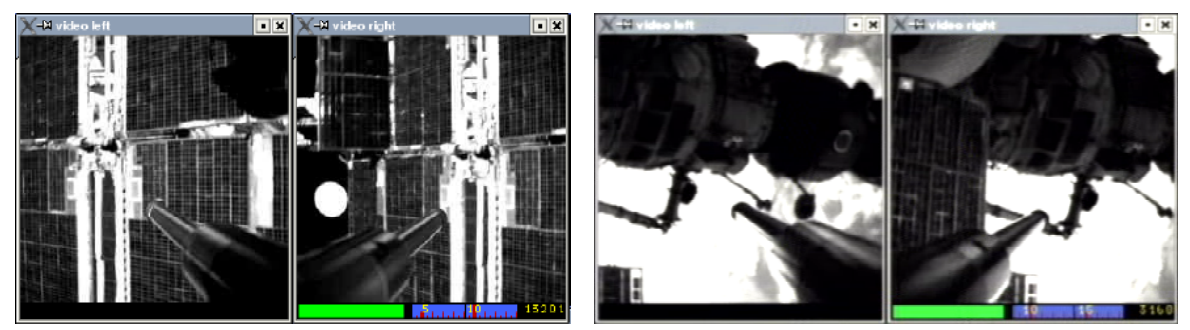

Fig. 9. Stereo video images taken by the integrated cameras of ROKVISS showing on left the arm pointing at the solar panel, and at the right pointing to the Soyuz docked to the ISS.

tification procedure based upon Recursive Least Squares algorithms is used for identification. To investigate how the space environment affects the behavior of two interacting bodies, the ROKVISS experiment contour is used to measure the energy dissipation occurring during intermittent impact events, as well as the frictional forces acting between two bodies while they are moving w.r.t. each other in a lasting contact situation.

The telerobotic concept of ROKVISS is based on DLR-RM's shared control/shared local autonomy techniques (see Sec. 3.1) as introduced with ROTEX and improved as MARCO supervisory control concept within GETEX [10]: All predefined tasks of ROKVISS can be executed by sending a path or a force trajectory to the on-board system. Feedback to the manmachine-interface (MMI) of the MARCO telerobotic ground control station is provided via the on-board camera system and the system's housekeeping data. Due to limited direct link experiment time the following automatic mode experiments have been performed (independent of direct radio link): Predefined trajectories (a) without force contact, (b) with force contact (i.e. contour tracking or movement against spring load) and (c) with a change from noncontact to contact condition (contact dynamics experiment). In contrast to the automatic mode experiments, the teleoperation experiments are conducted via direct operator interaction (see ROTEX 3.1) instead of time-line-based mission activation.

Another important issue of ROKVISS is to verify a high-fidelity forcereflecting telemanipulation concept based on the dedicated high-speed direct radio link to show the feasibility of immersive telepresence methods for future satellite servicing tasks. Differently to the teleoperation mode the human operator is "immersively" included into the control loop consuming stereo video images (like Fig. 9) in conjunction with the measured robot joint/torque values, which are fed back to the MMI on ground (see Fig. 10). The ROKVISS robot is controlled by the human operator using DLR-RM's force-feedback joystick to generate force and position commands, to drive the robot joints into the desired state. To cope with longer and varying time-delays, sophisticated bilateral control schemes have to be used. Among common bilateral 


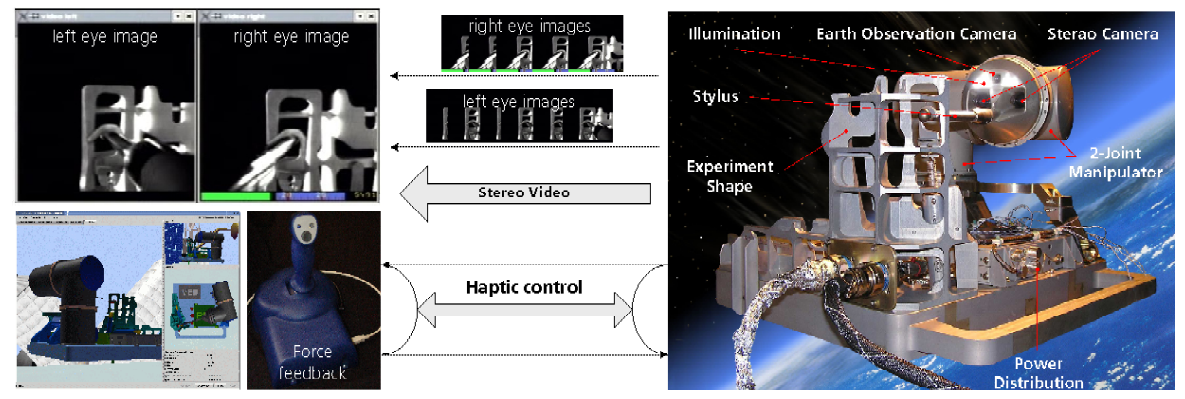

Fig. 10. High-fidelity telepresence control: During direct radio link contact the ROKVISS manipulator can be commanded by an operator on ground. A stereo camera is mounted on the 2nd joint: The stereo video images, together with the current robot joint and torque values, are fed back in real-time to an operator at the ground station where the operator controls the manipulator via a force-feedbackcontrol device.

control schemes new approaches as time domain passivity and wave-variables, in which the time-varying delay is simulated and compensated due to the orbit of the ISS, have been developed to investigate their efficiency and feasibility under real mission conditions $[7,28,29,30]$.

\subsection{DLR's Telerobotics Technologies for On-Orbit Servicing}

The experience gained with ROTEX laid an excellent basis for projects and studies on space robotics. ROTEX and ETS-VII were pure experimental systems to show and improve the principles of ground control under time delay constraints. The ROKVISS technology experiment investigates innovative advances in space robotics and demonstrates current capabilities in stateof-the-art A\&R technology, demonstrating the principle readiness of space robots for On-Orbit-Servicing applications. As the previous space robotics experiments has shown, the performance needed by telemanipulation requires a hierarchically and modularly structured shared automation concept tunable to the special operational case, which in addition allows human interference on different levels of supervisory and decision control [18, 20, 23].

\section{DLR's Light-Weight Robot Technology}

Service robotics requires robots which are able to manipulate objects in an unknown, changing environment, in direct interaction with humans as well as fully autonomously, as far as possible. Basic requirements have to be met by the arms and hands, which differ substantially from industrial manipulators: Space robotics as well as service robotic applications require light weight arms for safety reasons and human friendly interaction as well as to enable mobility. A load to weight ratio of 1:1 similar to that of the human arm is desired. 


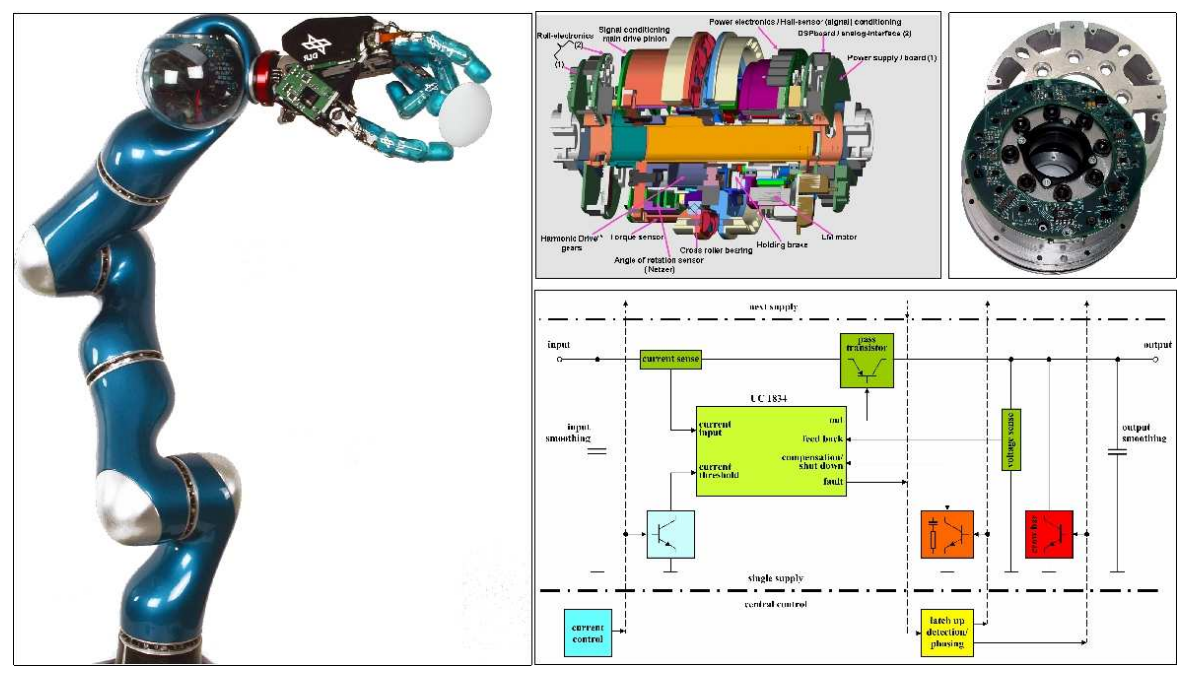

Fig. 11. The DLR's operational light-weight robot technology (left) is based on a modular drive concept (top left) with integrated torque sensors (top right); The latch-up protection power supply circuit (bottom) has been designed and integrated into the robotics joint electronics for space applicability.

Interaction with unknown environments needs compliant arms and fingers, facilitated by the information of many different sensors and advanced control strategies. To integrate different multi-sensory components as arms, hands, and maybe mobile platforms into mobile robotics applications, sophisticated mechatronic concepts and an adequate, flexible architecture of the control system are needed. To provide arm and hand systems that match these requirements is one goal of a long term project at DLR-RM. Especially, the last years, our focus on space robotics was caused by strong considerations, how to push robotic technologies towards space servicing applications. We developed a new generation of light weight robots with an unbeatable weight to load ratio as well as impressive control features, which make the system easy-to-use and safe for space as well as terrestrial servicing applications [34, 31, 17].

The design philosophy of DLR's light-weight-robots was to achieve a type of manipulator similar to the kinematic redundancy of the human arm, a load to weight ratio of better than $1: 2$, a total system-weight of less than $20 \mathrm{~kg}$ for arms with a reach space of up to $1,5 \mathrm{~m}$, no bulky wiring on the robot (and no electronics cabinet as it comes with every industrial robot), and a high dynamic performance. As all modern robot control approaches are based on commanding joint torques, joint torque control allowing programmable impedance, stiffness and damping, was a must for us. Also, the use of precise motor position sensing and link angular sensing has been considered as absolutely essential. 
The new robot arm concept (see Fig. 11) aimed at a completely modular assembly system with only a few basic components concerning joint mechanics, electronics and links. It is based on a fully modular joint-link-assembly system, with only a few basic components, namely three one-dof robot jointlink types and a two-dof wrist joint. This modularity concept was supported by SIMPACK, a powerful kinematics-dynamics analysis and design software. In general the modularity concept gives a number of advantages, e.g. rotation symmetric components, few single parts, short force transmission from bearing to off-drive connection, identical design for pitch and roll joints, big hollow shaft in all joints with up to $30 \mathrm{~mm}$ diameter, enabling the placement of cables and plug links inside the arm.

In addition to the mechanical modularity, also the robot electronics was designed in a modular manner. Each joint unit contains the power electronics for the motor and a motor current controller board, analog and digital signal processing hardware for the sensor signals and a DSP board for decentralized joint control. A DC-DC converter board provides the different voltages required by the joint electronics. A high-speed optical serial bus (SERCOS) is connecting the joints with the central computer. The only additional external connections are the power supply wires for the electronics and the motor.

\section{Telerobotic Control and Telemanipulation Concept}

Satellite repair in LEO or GEO should be performed under direct control of a human operator, due to the fact, that such missions can not be prepared in detail off-line on-ground. On the other hand, planetary exploration missions, must be performed by a task-directed programming and control system, which requires intelligent sensor processing methods, sophisticated planning algorithms, and robust FDIR (failure detection, isolation and recovery) techniques.

DLR has developed MARCO (Modular Automation and Robotics Controller architecture) [9], which integrates both worlds, direct telemanipulation as well as off-line programming combined with features for autonomous execution. The goal for the development this high-level programming system was to design a unified concept for a flexible, highly interactive, on-line programmable teleoperation station as well as an off-line programming tool, which includes all the sensor-based control features as tested already in ROTEX [14], but in addition provides the possibility to program a robot system on an implicit, task-directed level.

For that reason MARCO is based on a 2in2-layer-concept, which represents the hierarchical control structure from the planning to the executive layer: On the implicit (Payload Expert) level the instruction set is reduced to what has to be done. No specific robot actions will be considered at this task-oriented level. On the other hand the robot system has to know how the task can be successfully executed, which is described in the explicit (Robotic Expert) control layers. 

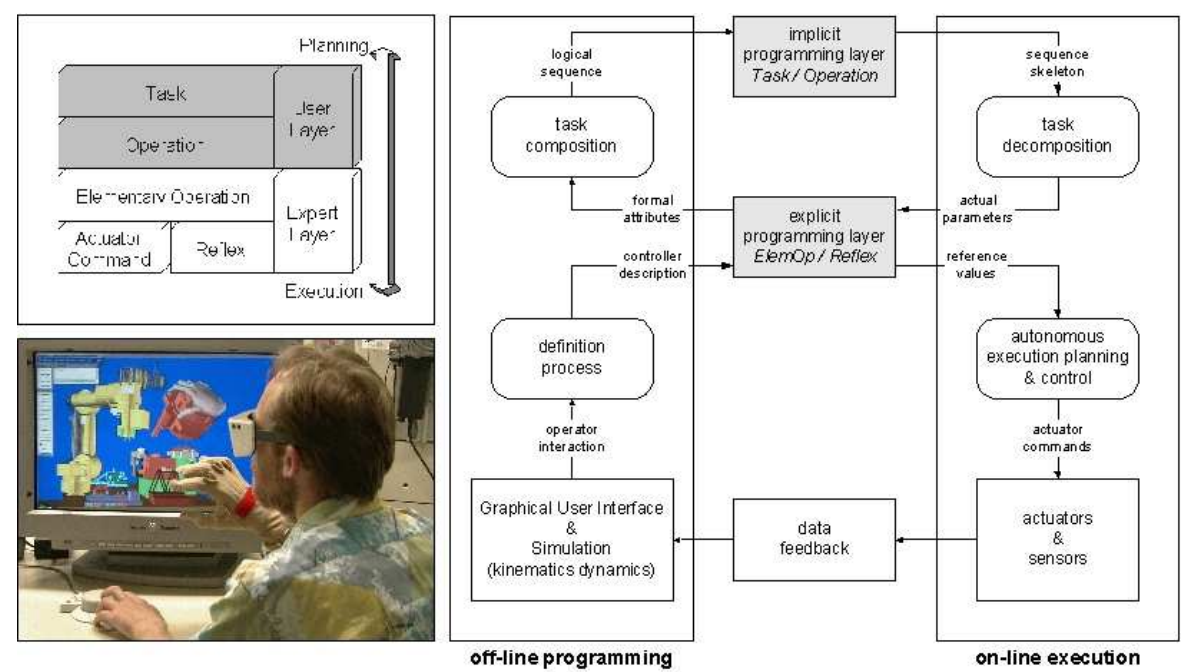

Fig. 12. The programming and execution layers of MARCO.

\section{DLR's Envisaged On-Orbit Servicing Applications}

The previous orbital telerobotic experiments have evaluated and demonstrated key robotics technologies for prototypical On-Orbit Servicing tasks. For real On-Orbit-Servicing as a business case the feasibility of these technologies have to be demonstrated first by realistic most common servicing application scenarios as extending the ageing of a functional satellite or repairing a malfunctioned one.

\subsection{TECSAS - Technology Demonstration for On-Orbit Servicing}

A German-Russian space robotics project is going on: the TECSAS (TEChnology SAtellite for demonstration and verification of Space systems) project aims at the in-orbit qualification of the key robotics elements (both hardware and software) for advanced space maintenance and servicing system, especially w.r.t. docking and robot-based capturing procedures. It is planned, in close cooperation with the Russian Babakin Space Center, to perform such sensitive operations as rendezvous and close approach maneuvers, which will be necessary for further servicing activities. The Russian Multi-Purposes Orbital Boost Platform as the base module for the mission provides the insertion into initial parking orbit as well as supports rendezvous and docking maneuvers. For docking and capturing operations we prefer to use our own robotics means (manipulator, controller, etc.) as well as MARCO as the ground control environment.

The entire mission will be performed utilizing following steps: far rendezvous, close approach, inspection fly around, formation flight, capture, 

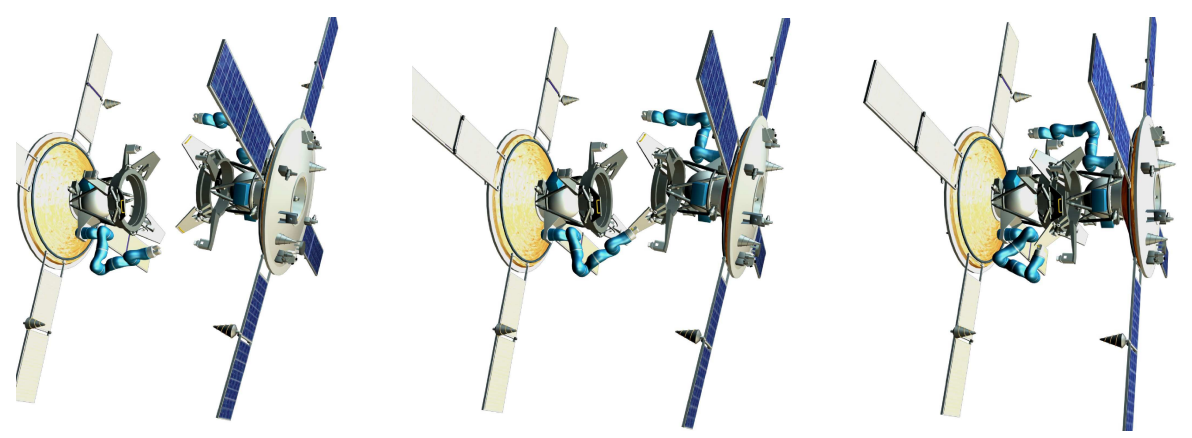

Fig. 13. The capturing approach of the floating, non-cooperative target system: (1) Close approach phase, (2) capture of the target by chaser with the aid of manipulator, and (3) pulling along guide elements of Docking Units and fixation with the help of locks of Docking Units.

stabilization and calibration of the compound, compound flight maneuver, manipulation of the target, active ground control via telepresence, passive ground control during autonomous operations (monitoring), and controlled de-orbiting of the compound. For the capturing of the floating target satellite, the control modes will be applied as developed in the ESS study and verified during the ETS-VII mission. After capturing, for compound stabilization, the manipulator can be used as an active damping system: since the platform, including the robot system, and the captured target system build one compound system, the dynamic behavior of the complete system can be influenced by moving the robot. Also for de-orbiting maneuvers the manipulator can be used as a support system: it can be considered as a passive link building the mechanical interface between the chaser and the target system. The geometry of the compound system can be influenced by the robot's attitude. Additionally, the robot can be considered as an active link, thus controlling the thrusters vector pointing to the common center of mass.

\subsection{CX-OLEV - Towards On-Orbit-Servicing as Business Case}

Similar to the TECSAS experiment, where we give the priority to the technological aspects of space robot servicing tasks, we will create the first business case in on-orbit-servicing, in a strong cooperation with the industry: attaching a "tugboat" to a satellite, whose propellant is declining, the lifetime of valuable telecommunication satellites could be prolonged for several years. Telecommunications satellites typically cost at least $\$ 250$ million - and they are designed for an average useful on-orbit life of 10-15 years. Once their onboard propellant load is depleted, the satellites are boosted into a disposal orbit and decommissioned, even though their revenue-generating communications relay payloads continue to function. 

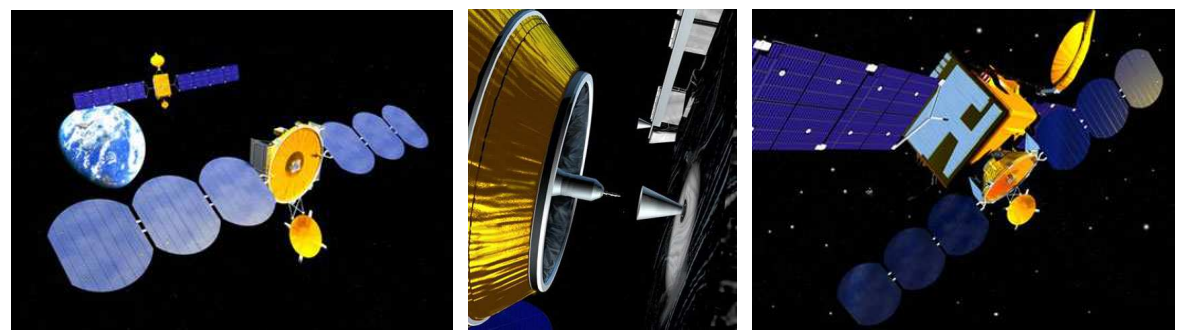

Fig. 14. The capturing approach of a floating, cooperative target system: (1) Far approach phase, (2) close approach phase with a relative motion of zero between the target and the servicer, and (3) compound satellite system after docking.

Our industrial partner Orbital Recovery Corp. has initiated its ConeXpressOrbital Live Extension System (CX-OLEV) which will significantly prolong the operating lifetimes of these valuable telecommunications satellites. The CX-OLEV system will operate as an orbital tugboat, supplying the propulsion, navigation and guidance to keep a telecommunications satellite in its proper orbital slot for many additional years. Another application of CXOLEV could be the rescue of a spacecraft that have been placed in a wrong orbit by their launch vehicles, or which have become stranded in an incorrect orbital location during positioning maneuvers. The system is designed to easily mate with all telecommunications satellites now in space or on the drawing boards.

It will rendezvous with the telecommunications satellite and link up using our proprietary docking device that connects to the telecommunication satellite's apogee kick motor, as we have proposed within the ESS technology study (see Sec. 3.3). Apogee kick motors are used by nearly every telecommunications satellite for orbital boost and station-keeping, and they provide a strong, easily accessible interface point for the CX-OLEV linkup that is always within the satellite's center of gravity. DLR-RM's contributions to the CX-OLEV system are closely related to the experiences gained during previous telerobotic experiments:

- to designe and develop a Capture Tool, including locking mechanism, sensors and control software, similiar to the ESS capture tool (see Sec. 3.3);

- to provide a telemanipulation software to guide the Capture Tool, which is mounted to the CX-OLEV system, into the apogee-motor of the target satellite;

- to deploy and perform the Ground Control System and capture strategies. 


\section{Conclusion}

As previous pioneering telerobotic experiments has been shown, the achievements and deployments in Automation and Robotics (A\&R) during the last 20 years reach a profession which can be used in near future to support, to unburden, even to replace the human by safe critical or monotonous work as a matter of routine. It seems that in future space robotics will become a key technology for the exploration of outer space and the operation and maintenance of space stations, satellites and other platforms, saving costs and relieving man from dangerous tasks. Within future spaceflights and on-orbit servicing missions intelligent robotics technology will be used for assisting in and carrying out different tasks inside spacecrafts or space laboratories (internal usage) as well as in free space (external usage). But we do not have to wait until robots are really autonomous or intelligent, since by modern teleoperation and telepresence we are able to remotely control robot systems from the ground in the sense of "prolonging man's arm into space".

Ongoing development of space robotics technology has to focus on intelligent, sensor-controlled, light-weight robots using modular, flexible grippers, articulated hands and tool systems for high versatility. Even improved manmachine interfaces for teleoperation and supervisory control concepts has to be deployed for efficient cooperation and coordination of multi-arm and multirobot system. But in particular, concerning the manipulative skills present hardware and software concepts requested to cover the range from high-fidelity telepresent manipulation up to (partly) autonomous operation is still available for the step towards the first business case in On-Orbit-Servicing.

\section{References}

1. S. Hayati, S.T. Venkataraman (1989) Design and Implementation of a Robot Control System with Traded and Shared Control Capability. In: Proc. of the IEEE Int. Conf. on Robotics and Automation (ICRA), 3:1310-1315

2. L. Conway, R. Volz, M. Walker (1987) Tele-Autonomous Systems: Methods and Architectures for Intermingling Autonomous and Telerobotic Technology. In: Proc. of the IEEE Int. Conf. on Robotics and Automation (ICRA), 2:11211130

3. D. King (2002) On-orbit servicing business; an industry vision. In: Proc. of the 1st Bilateral DLR-CSA Workshop on On-Orbit Servicing of Space Infrastructure Elements via Automation \& Robotics Technologies (OOS 2002) "Defining a Way Forward", Cologne, Germany

4. A. Ellery (2002) Handling technology: technology of robotic in-orbit servicing. In: Proc. of the 1st Bilateral DLR-CSA Workshop on On-Orbit Servicing of Space Infrastructure Elements via Automation \& Robotics Technologies (OOS 2002) "Defining a Way Forward", Cologne, Germany

5. J.-C. Piedboeuf (2002) On-Orbit Servicing and Beyond: A Canadian Perspective. In:, Proc. of the 1st Bilateral DLR-CSA Workshop on On-Orbit Servic- 
ing of Space Infrastructure Elements via Automation \& Robotics Technologies (OOS 2002) "Defining a Way Forward", Cologne, Germany

6. J. Andary, P. Spidaliere (1993) Development test flight of the Flight Telerobotic Servicer: design description and lessons learned. In: Proc. of the IEEE Int. Conf. on Robotics and Automation (ICRA), 2:1121-1130

7. J. Artigas, C. Preusche, G. Hirzinger (2004) Wave Variables based Bilateral Control with a Time Delay Model for Space Robot Applications. In: Robotik 2004, VDI-Bericht, München, Germany, 1841:101-108

8. B. Brunner, K. Arbter, G. Hirzinger (1994a) Task Directed Programming of Sensor Based Robots. In: Proc. of the IEEE/RSJ Int. Conf. on Intelligent Robots and Systems (IROS), 2:1080-1087

9. B. Brunner, K. Landzettel, B.-M. Steinmetz, G. Hirzinger (1995) Tele-SensorProgramming - A task-directed programming approach for sensor-based space robots. In: ICAR - The 7th International Conference on Advanced Robotics, Sant Feliu de Guixols, Catalonia (Spain)

10. B. Brunner, K. Landzettel, G. Schreiber, B.-M. Steinmetz, G. Hirzinger (1999) A universal Task-Level Ground Control and Programming System for Space Robot Applications - The MARCO Concept and its Application to the ETSVII Project. In: Proc. of the 5th International Symposium on Artificial Intelligence, Robotics and Automation in Space (iSAIRAS), ESTEC, Noordwijk, The Netherlands, 507-514

11. G. Hirzinger, K. Landzettel, J. Heindl, J. Dietrich (1994) ROTEX - The First Robot in Space. In: The 5th European Symposium on Space Environmental Control Systems and 24th International Conference on Environmental Systems (ICES), Friedrichshafen, Germany

12. Ch. Fragerer, G. Hirzinger (1992) Predicitve Telerobotic Concept for Grasping a Floating Object. In: International Federation of Automatic Control, Spacecraft Automation and On-Board Autonomous Mission Control, Darmstadt, Germany

13. G. Hirzinger, B. Brunner, J. Dietrich, J. Heindl (1993) Sensor-Based Space Robotics - ROTEX and Its Telerobotic Features. In: Proc. of the IEEE Int. Conf. on Robotics and Automation (ICRA), 9:649-663

14. G. Hirzinger (1993) Multisensory Shared Autonomy and Tele-SensorProgramming - Key Issuses in Space Robotics. In: IAS - 3 International Conference in Intelligent Autonomous Systems, Pittsburgh, PA, USA

15. G. Hirzinger, K. Landzettel, Ch. Fagerer (1994) Telerobotics with large time delays - the ROTEX experience. In: Proc. of the IEEE/RSJ Int. Conf. on Intelligent Robots and Systems (IROS), München, Germany

16. E. Freund, J. Rossmann (2000) Space Robot Commanding and Supervision by means of Projective Virtual Reality: The ERA Experiences. In: Proc. of the 7th Conference on Telemanipulator and Telepresence Technologies, 312-322

17. G. Hirzinger, K. Landzettel, D. Reintsema, C. Preusche, A. Albu-Schäffer, B. Rebele, M. Turk (2005) ROKVISS - Robotics Component Verification on ISS. In: Proc. of the 8th International Symposium on Artificial Intelligence, Robotics and Automation in Space (iSAIRAS), München, Germany

18. G. Hirzinger, B. Brunner, R. Lampariello, K. Landzettel, J. Schott, B.-M. Steinmetz (2000) Advances in Orbital Robotics. In: Proc. of the IEEE Int. Conf. on Robotics and Automation (ICRA), 898-907

19. G. Hirzinger, B. Brunner, R. Lampariello, K. Landzettel, G. Schreiber et al. (2000) A Unified Ground Control and Programming Methodology for Space 
Robotics Applications - Demonstrations on ETS-VII. In: Proc. of the International Symposium on Robotics (ISR 2000), 422-427

20. G. Hirzinger, K. Landzettel, B. Brunner, M. Fischer, C. Preusche, D. Reintsema, A. Albu-Schäffer, G. Schreiber, B.M. Steinmetz (2004) DLR's Robotics Technologies for On-Orbit Servicing. In: Advanced Robotics, Special Issue on Service Robots in Space (1), 18:139-174

21. R. Lampariello, G. Hirzinger (2000) Freeflying Robots -Inertial Parameters Identification and Control Strategies. In: Proc. of ASTRA 2000 - The 6th ESA Workshop on Advanced Space Technologies for Robotics and Automation, Noordwijk, The Netherlands

22. G. Hirzinger, K. Landzettel, B. Brunner, I. Schaefer, M. Fischer et al. (1999) DLR's Robotics Lab - Recent developements in Space Robotics. In: Proc. of the 5th International Symposium on Artificial Intelligence, Robotics and Automation (iSAIRAS), Noordwijk, The Netherlands

23. K. Landzettel, B. Brunner, R. Lampariello, C. Preusche, D. Reintsema, G. Hirzinger (2004) System Prerequisites and Operational Modes for On-OrbitServicing. In: The International Symposium on Space Technology and Science (ISTS), Miyazaki, Japan

24. K. Landzettel, B. Brunner, K. Deutrich, G. Hirzinger, G. Schreiber, B.-M. Steinmetz () DLR's Experiments on the ETS VII Space Robot Mission. In: Proc. of the 9th International Conference on Advanced Robotics (ICAR), Tokyo, Japan

25. R. Lampariello, S. Agrawal, G. Hirzinger (2003) Optimal Motion Planning of Free-Flying Robots. In: Proc. of the IEEE Int. Conf. on Robotics and Automation (ICRA),

26. B. Rebele, R. Krenn, B. Schäfer (2002) Grasping Strategies and Dynamic Aspects in Satellite Capturing by Robotic Manipulator. In: Proc. of ASTRA 2002 - The 7th ESA Workshop on Advanced Space Technologies for Robotics and Automation, Noordwijk, The Netherlands

27. K. Arbter, J. Langwald, G. Hirzinger, G.Q. Wei, P. Wunsch (1998) Proven Techniques for Robust Visual Servo Control. In: Proc. of the IEEE Int. Conf. on Robotics and Automation (ICRA),

28. B. Hannaford, J.H. Ryu (2002) Time Domain Passivity Control of Haptic Interfaces. In: IEEE - Transactions on Robotics and Automation, 18:1-10

29. J.H. Ryu, B. Hannaford, C. Preusche, G. Hirzinger (2003) Time Domain Passivity Control with Reference Energy Behavior. In: Proc. of the IEEE/RSJ Int. Conf. on Intelligent Robots and Systems (IROS), 3:2932-2937

30. C. Preusche, G. Hirzinger, J.H. Ryu, B. Hannaford (2003) Time Domain Passivity Control for 6 Degrees of Freedom Haptic Displays. In: Proc. of the IEEE/RSJ Int. Conf. on Intelligent Robots and Systems (IROS), 3:2944-2949

31. G. Hirzinger, N. Sporer, A. Albu-Schäffer, M. Hähnle, R. Krenn, A. Pascucci, M. Schedl (2002) DLR's torque-controlled light weight robot III - are we reaching the technological limits now? In: Proc. of the IEEE Int. Conf. on Robotics and Automation (ICRA), 1710 - 1716

32. G. Hirzinger, N. Sporer, M. Schedl, J. Butterfaß, M. Grebenstein (2002) Robotics and Mechatronics in Aerospace. In: The 7th International Workshop on Advanced Motion Control (AMC), 19-27

33. G. Hirzinger, B. Brunner, J. Dietrich, J. Heindl (1994) ROTEX - The First Remotely Controlled Robot in Space. In: Proc. of the IEEE Int. Conf. on Robotics and Automation (ICRA), 3:2604-2611 
34. G. Hirzinger, J. Butterfaß, M. Grebenstein, I. Schaefer, N. Sporer, M. Fischer, H. Liu, A. Albu-Schäffer, M. Schedl, P. Neumann (2001) Space Robotics - Driver for a new mechatronic Generation of light-weight arms and multifingered hands. In: Proc. of the IEEE/ASME International Conference on Advanced Intelligent Mechatronics (AIM), 1160 - 1168

35. G. Hirzinger, K. Landzettel, B. Brunner, M. Fischer, C. Preusche, D. Reintsema, A. Albu-Schäffer, G. Schreiber, B.-M. Steinmetz (2004) DLR's robotics technologies for on-orbit servicing. In: Advanced Robotics, RSJ - The International Journal of the Robotics Society of Japan, 18(2):139-174. VSP, Netherlands, ISSN 0169-1864

36. B. Schäfer, M. Lösch (1999) Simulation of Elastic Space Manipulator Dynamics During Satellite Capturing. In: Proc. of the 4th International Conference of the European Association for Structural Dynamics (EURODYN '99), Prague, Czech Republic

37. B. Schäfer, M. Lösch, K. Landzettel (1998) Simulation of Manipulator Deployment and Satellite Capturing Dynamics. In: Proc. of the 5th ESA Workshop on Advanced Space Technologies for Robotics and Automation (ASTRA 98), Noordwijk, The Netherlands

38. R.W Longman, R.E. Lindberg, M.F. Zedd (1987) Satellite-mounted Robot Manipulators - New kinematic and Reaction Moment Compensation. In: International Journal of Robotics Research (3)

39. S. Dubowsky, E. Papadopoulos (1993) The Kinematics, Dynamics and Control of Free-Flying and Free-Floating Space Robotic Systems. In: IEEE Transactions on Robotics and Automation (5)

40. H.G. McCain, J.F. Andary (1988) The Flight Telerobotic Servicer Project and systems overview. In: Electronics and Aerospace Conference, 18(2):139-174

41. M. Oda, D. Toshitsugu (1997) Teleoperation System of ETS-VII Robot Experiment Satellite. In: Proc. of the IEEE/RSJ Int. Conf. on Intelligent Robots and Systems (IROS), 3:1644-50

42. M. Oda, T. Doi, K. Wakata (2001) Tele-manipulation of a satellite mounted robot by an on-ground astronaut. In: Proc. of the IEEE Int. Conf. on Robotics and Automation (ICRA), 2:1891-96

43. M. Oda (1999) Space Robot Experiments on NASDAs ETS-VII Satellite. In: Proc. of the IEEE Int. Conf. on Robotics and Automation (ICRA), 2:13901395

44. W.-K. Yoon, T. Goshozono, H. Kawabe, M. Kinami, Y. Tsumaki, M. Uchiyama, M. Oda, T. Doi (2001) Model-based teleoperation of a Space robot on ETS-VII using a haptic interface. In: Proc. of the IEEE Int. Conf. on Robotics and Automation (ICRA), 1:407-12

45. I. Kawano, M. Mokuno, et. al. (2001) Result of Autonomous Rendezvous Docking Experiment of Engineering Test Satellite VII. In: Journal of Spacecraft and Rockets, 38(1):105-111

46. I. Kawano, M. Mokuno, T. Miyano, T. Suzuki (2000) Analysis and Evaluation of GPS Relative Navigation Using Carrier Phase for RVD Experiment Satellite of ETS-VII. In: ION GPS 2000 - The 13th International Technical Meeting of the Satellite Division of the Institute of Navigation, 1655-60

47. G. Visentin, F. Didot (1999) Testing Space Robotics on the Japanese ETS-VII Satellite. In: ESA Bulletin, 99:61-65 
48. I. Kawano, et. al. (1998) First Results of Autonomous Rendezvous Docking Experiments on NASDA's ETS-VII Satellite. In: Proceedings of the 49th International Astronautical Congress, Melbourne, Australia

49. Y. Fukushima, N. Inaba, M. Oda (2000) Capture and berthing experiment of a massive object using ETS-VII's space robot - World's first on-orbit satellite capture experiment by space robot system. In: Proceedings of AIAA/AAS Astrodynamics Specialist Conference, Denver, CO

50. K. Yoshida, K. Hashizume, S. Abiko (2001) Zero Reaction Maneuver: Flight Validation with ETS-VII Space Robot and Extension to Kinematically Redundant Arm. In: Proc. of the IEEE Int. Conf. on Robotics and Automation (ICRA), 1:441-46

51. T. Imaida, Y. Yokokohji, T. Doi, M. Oda, T. Yoshikawa (2001) GroundSpace Bilateral Teleoperation Experiment Using ETS-VII Robot Arm with Direct Kinesthetic Coupling. In: Proc. of the IEEE Int. Conf. on Robotics and Automation (ICRA), 1:1031-1038

52. Y. Yokokohji, T. Imaida, Y. Iida, T. Doi, M. Oda, T. Yoshikawa (2000) Bilateral Teleoperation: Towards Fine Manipulation with Large Time Delay. In: (ISER), $11-20$ 